\title{
SOME REMARKS ON THE SCHRÖDINGER EQUATION WITH A POTENTIAL IN $L_{t}^{r} L_{x}^{s}$
}

\author{
Piero D'Ancona \\ Università di Roma "La Sapienza" \\ Dipartimento di Matematica, \\ Piazzale A. Moro 2, I-00185 Roma, Italy, \\ email: dancona@mat.uniroma1.it \\ tel/fax: +39-06-4991-3092 \\ Vittoria Pierfelice and Nicola Visciglia \\ Università di Pisa \\ Dipartimento di Matematica \\ Via F. Buonarroti 2, I-56127 Pisa, Italy \\ email: pierfelice@dm.unipi.it,viscigli@mail.dm.unipi.it
}

\begin{abstract}
We study the dispersive properties of the linear Schrödinger equation with a timedependent potential $V(t, x)$. We show that an appropriate integrability condition in space and time on $V$, i.e. the boundedness of a suitable $L_{t}^{r} L_{x}^{s}$ norm, is sufficient to prove the full set of Strichartz estimates. We also construct several counterexamples which show that our assumptions are optimal, both for local and for global Strichartz estimates, in the class of large unsigned potentials $V \in L_{t}^{r} L_{x}^{s}$.
\end{abstract}

\section{INTRODUCTION}

Dispersive properties of evolution equations have become in recent years a crucial tool in the study of a variety of questions, including local and global existence for nonlinear equations, well posedness in Sobolev spaces of low order, scattering theory and many others. In particular, the free Schrödinger equation

$$
i \partial_{t} u-\Delta u=0
$$

exhibits a rich set of dispersive and smoothing properties. The basic ones are the $L^{p}-L^{q}$ estimates

$$
\|u(t, \cdot)\|_{L^{q}} \leq \frac{C}{t^{\sigma}}\|u(0, \cdot)\|_{L^{p}}, \quad \frac{1}{p}+\frac{1}{q}=1, \quad p \in[1,2], \quad \sigma=\frac{n}{2}-\frac{n}{q},
$$

and the Strichartz estimates, which we shall recall shortly (see Definition 1.1 below).

The usual route to prove these estimates is: first deduce the $L^{p}-L^{q}$ estimates from the explicit expression of the fundamental solution; then, use them to derive the Strichartz estimates via suitable functional analytic arguments. Standard references on the subject are [7] and [12; see also [5]. However, in more general situations this approach is not feasible, and indeed, for some generalizations of the Schrödinger equation, Strichartz estimates may hold even when pointwise estimates fail (see e.g. 11, 2], 4). Thus it appears that Strichartz estimates have a more fundamental nature and a greater generality.

Date: November 9, 2018.

2000 Mathematics Subject Classification. 35B40, 35B25, 35B65, 35Q40, 35 Q55.

Key words and phrases. Schrödinger equation, time-dependent potential, Strichartz estimates.

Support. The authors are partially supported by the Research Training Network (RTN) HYKE and by grant HPRN-CT-2002-00282 from the European Union. The third author is supported also by INDAM. 
Here we shall focus on the Schrödinger equation with a time-dependent potential

$$
i \partial_{t} u-\Delta u+V(t, x) u=0
$$

and its inhomogeneous version with a source term. The great interest of this equation, both from the physical and from the mathematical point of view, is well known. Many results on the dispersive properties are available when the potential $V=V(x)$ depends only on space variables (see, among others, 9], 11, 15] and the references therein). On the other hand, the time-dependent case is much more delicate; almost all available results are of a perturbative nature, requiring some smallness of the potential $V(t, x)$ (see [9], 15, and, for small potentials of very low regularity, [8], [13]; concerning the case of time-periodic potentials, see [10], and [16]; see also [6]).

Our goal here is to show that, by purely elementary arguments based on integrability properties of the potential (as opposed to the "global" smallness required in the above mentioned results), it is possible to obtain a great deal of information on the behaviour of the solution, and to prove the Strichartz estimates for a wide class of large potentials with no definite sign. Of course, the usual obstructions are present also in this general situation: existence of standing waves, rescaling and pseduoconformal symmetry of the equation. Using these, we are able to show that our conditions are also necessary, at least in the class of potentials under consideration.

Let us recall the classical Strichartz estimates for the Schrödinger equation, and introduce some notations. We use a prime to denote conjugate indices; moreover, for any subinterval $I$ of $\mathbb{R}$ (bounded or unbounded) we define the mixed space-time norms

$$
\|u\|_{L_{I}^{p} L^{q}} \equiv\left(\int_{I}\|u(t, \cdot)\|_{L^{q\left(\mathbb{R}^{n}\right)}}^{p} d t\right)^{1 / p}
$$

and when $I=\left[0,+\infty\left[\right.\right.$ we write simply $L^{p} L^{q}$ i n place of $L_{I}^{p} L^{q}$. Similarly, we shall write

$$
C_{I} L^{p} \equiv C\left(I ; L^{p}\right), \quad C L^{p} \equiv C\left(\left[0,+\infty\left[; L^{p}\right)\right.\right.
$$

for $1 \leq p \leq \infty$.

Definition 1.1. Let $n \geq 2$. The pair $(p, q)$ is said to be (Schrödinger) admissible if

$$
\frac{1}{p}+\frac{n}{2 q}=\frac{n}{4}, \quad p, q \in[2, \infty], \quad(n, p, q) \neq(2,2, \infty) .
$$

The Strichartz estimates can be stated as follows: for all admissible couples $(p, q)$ and $(\tilde{p}, \tilde{q})$ there exists a constant $C(p, \tilde{p})$ such that, for all interval $I \subseteq \mathbb{R}$ (bounded or unbounded), for all functions $u_{0}(x) \in L^{2}\left(\mathbb{R}^{n}\right)$, and $F(t, x) \in L_{I}^{\tilde{p}^{\prime}} L^{\tilde{q}^{\prime}}$ the following inequalities hold:

$$
\begin{gathered}
\left\|e^{i t \Delta} u_{0}\right\|_{L_{I}^{p} L^{q}} \leq C(p, \tilde{p})\left\|u_{0}\right\|_{L^{2}} \\
\left\|\int_{0}^{t} e^{i(t-s) \Delta} F(s) d s\right\|_{L_{I}^{p} L^{q}} \leq C(p, \tilde{p})\|F\|_{L_{I}^{\tilde{p}^{\prime}} L^{\tilde{q}^{\prime}}}
\end{gathered}
$$

Note that the constant is independent of the interval $I$.

Clearly, when $n \geq 3$ the constant can be taken also independent of $p$ and $\tilde{p}$ : we shall denote this universal constant (which depends now only on the space dimension $n$ ) by $C_{0}$. When $n=2$, the constant is unbounded as $p \downarrow 2$ or $\tilde{p} \downarrow 2$.

Here $e^{i t \Delta}$ is the unitary operator

$$
e^{i t \Delta} f=\int_{\mathbb{R}^{n}} \frac{e^{-\frac{i|x-y|^{2}}{4 t}}}{(4 \pi i t)^{n / 2}} f(y) d y, \quad \int_{0}^{t} e^{i(t-s) \Delta} F(s) d s=\int_{0}^{t} \int_{\mathbb{R}^{n}} \frac{e^{-\frac{i|x-y|^{2}}{4(t-s)}}}{(4 \pi i(t-s))^{n / 2}} F(s, y) d y d s
$$

which is properly defined on $L^{2}$ but can be extended to 
different $L^{p}$ spaces using e.g. these explicit expressions.

Consider now the differential equation

$$
i \partial_{t} u-\Delta u+V(t, x) u=F(t, x), \quad u(0, x)=u_{0}(x) .
$$

For low regularity solutions, it is customary to replace (1.10) with the integral equation

$$
u(t, x)=e^{i t \Delta} u_{0}(x)+\int_{0}^{t} e^{i(t-s) \Delta}[F(s)-V(s) u(s)] d s .
$$

The two formulations are equivalent under very mild assumptions on the class of solutions; we shall not discuss this problem here, instead we shall use the integral formulation exclusively.

We can now state our first result:

Theorem 1.1. Let $n \geq 2$, let $I$ be either the interval $[0, T]$ or $[0,+\infty[$, and assume $V(t, x)$ is a real valued potential belonging to

$$
V(t, x) \in L_{I}^{r} L^{s}, \quad \frac{1}{r}+\frac{n}{2 s}=1
$$

for some fixed $r \in[1, \infty[$ and $s \in] n / 2, \infty]$. Let $u_{0} \in L^{2}$ and $F \in L_{I}^{\tilde{p}^{\prime}} L^{\tilde{q}^{\prime}}$ for some admissible pair $\left(\tilde{p}^{\prime}, \tilde{q}^{\prime}\right)$.

Then the integral equation (1.11) has a unique solution $u \in C_{I} L^{2}$ which belongs to $L_{I}^{p} L^{q}$ for all admissible pairs $(p, q)$ and satisfies the Strichartz estimates

$$
\|u\|_{L_{I}^{p} L^{q}} \leq C_{V}\left\|u_{0}\right\|_{L^{2}}+C_{V}\|F\|_{L_{I}^{\tilde{p}^{\prime}} L^{\tilde{q}^{\prime}}}
$$

When $n \geq 3$, the constant $C_{V}$ can be estimated by $k\left(1+2 C_{0}\right)^{k}$, where $C_{0}$ is the Strichartz constant for the free equation, while $k$ is an integer such that the interval $I$ can be partitioned in $k$ subintervals $J$ with the property $\|V\|_{L_{J}^{r} L^{s}} \leq\left(2 C_{0}\right)^{-1}$. A similar statement holds when $n=2$, provided we replace $C_{0}$ by $C(p, \tilde{p})$.

Finally, when $F \equiv 0$ the solution satisfies the conservation of energy

$$
\|u(t)\|_{L^{2}} \equiv\left\|u_{0}\right\|_{L^{2}}, \quad t \in I .
$$

Remark 1.1. We emphasize that the potentials $V(t, x)$ considered in Theorem 1.1 may be both large and change sign. The usual smallness assumption is replaced here by the integrability condition with respect to time, which ensures smallness of $V$ on sufficiently small time intervals, and for $t>>1$.

Remark 1.2. By iterating the argument of the proof, it is easy to extend the above result to any potential of the form

$$
V=V_{1}+\cdots+V_{k}
$$

where $V_{1}, \ldots, V_{k}$ satisfy assumption (1.12), with possibly different indices $r_{j}, s_{j}$.

Remark 1.3. Note that when $I$ is a bounded interval, assumption (1.12) can be relaxed to

$$
V(t, x) \in L_{I}^{r} L^{s}, \quad \frac{1}{r}+\frac{n}{2 s} \leq 1 ;
$$

indeed, from (1.15), using Hölder's inequality in time we can easily show that also (1.12) holds, for a smaller value of $r$ and the same value of $s$.

Thus in particular we see that the existence part of our theorem extends a result of Yajima [17, who proved that the equation (1.11) (or (1.10) ) is well posed in $L^{2}$ with conservation of energy, provided the potential $V$ satisfies

$$
V=V_{1}+V_{2}, \quad V_{1} \in L_{I}^{r} L^{s}, \quad V_{2} \in L_{I}^{\infty} L^{\beta}
$$

with $\beta>1$ and

$$
\frac{1}{r}+\frac{n}{2 s}<1
$$


(see also the preceding remark).

When the potential $V(t, x)$ belongs to $L_{I}^{\infty} L^{n / 2}$, i.e., in the limiting case of Theorem 1.1 the result can not be true; indeed, this case includes the static potentials $V(x) \in L^{n / 2}$ without any positivity or smallness assumption. We mention that even for a nonnegative potential in $L^{n / 2}$ it is not known if the Strichartz estimates are valid in general. The best result in this direction is due to Rodnianski and Schlag 15 who considered bounded potentials defined on $\mathbb{R}^{n}$ satisfying the estimate $|V(x)| \leq C|x|^{-2-\epsilon}$ for $|x|$ large enough. However, in the limiting case we can prove a partial substitute of Theorem 1.1] To simplify our statement we introduce the following definition:

Definition 1.2. Let $V(x)$ be a real valued function such that

$$
H=\Delta-V(x)
$$

has a selfadjoint extension. We say that the potential $V(x)$ is of Strichartz type if for all bounded time interval $I=[0, T]$, for all $u_{0} \in L^{2}$ and $F \in L_{I}^{\tilde{p}^{\prime}} L^{\tilde{q}^{\prime}}$ with $(\tilde{p}, \tilde{q})$ admissible, the integral equation

$$
u(t, x)=e^{i t H} u_{0}+\int_{0}^{t} e^{i(t-s) H} F(s) d s
$$

has a unique solution $u(t, x) \in C_{I} L^{2}$ satisfying the Strichartz estimates

$$
\|u\|_{L_{I}^{a} L^{b}} \leq C(I, V)\left\|u_{0}\right\|_{L^{2}}+C(I, V)\|F\|_{L_{I}^{\tilde{p}^{\prime}} L^{\tilde{q}^{\prime}}}
$$

for all admissible pairs $(a, b)$.

Then we have:

Theorem 1.2. Let $n \geq 3$, let $I$ be a bounded interval $[0, T]$ and let $V(t, x) \in C_{I} L^{n / 2}$. Assume that for each $t \in I, V(t, \cdot)$ is of Strichartz type, while the functions $u_{0}$ and $F(t, x)$ are as in Theorem 1.1. Then the conclusion of Theorem 1.1 holds true (local Strichartz estimates).

The result holds also in the case $I=[0, \infty[$ (global Strichartz estimates) under the additional assumption: there exists $T_{0}>0$ such that $\|V(t, \cdot)\|_{L^{n / 2}} \leq\left(2 C_{0}\right)^{-1}$ for $t>T_{0}$.

Remark 1.4. By simple modifications in the proof, Theorem 1.2 can be extended to any potential of the form

$$
V(t, x)=V_{1}(t, x)+V_{2}(t, x),
$$

with $V_{1}$ as in the theorem while $V_{2} \in L_{I}^{\infty} L^{n / 2}$ satisfies

$$
\left\|V_{2}\right\|_{L_{I}^{\infty} L^{n / 2}} \leq \varepsilon\left(V_{1}\right)
$$

for a suitable small constant $\epsilon\left(V_{1}\right)$ depending only on $V_{1}$.

Example 1.1. To illustrate a possible use of Theorem 1.1 consider the semilinear Schrödinger equation

$$
i \partial_{t} u-\Delta u+f(u) u=0, \quad|f(u)| \leq C|u|^{\gamma}, \quad \gamma>1,
$$

$f$ real valued, which includes both focusing and defocusing equations with a power nonlinearity. Then we may regard (1.20) as a Schrödinger equation with a time dependent potential

$$
V(t, x)=f(u(t, x)) .
$$

We see that $V$ satisfies the assumptions of Theorem 1.1 provided

$$
u \in L^{a} L^{b}, \quad \frac{1}{a}+\frac{n}{2 b}=\frac{1}{\gamma}, \quad a<\infty .
$$

Thus any solution satisfying (1.21) satsfies the full set of Strichartz estimates.

For instance, in the case of the (focusing or defocusing) quintic Schrödinger equation in three dimensions, any solution $u \in L^{10} L^{10}$ satisfies the Strichartz estimates; this was the first step in the proof of the global well posedness for the radial defocusing three dimensional quintic in [3]. 
Example 1.2. We give a simple application of Theorem 1.2 Consider a real valued potential $V \in C L^{\frac{n}{2}}$ and assume it satisfies the bounds

$$
0 \leq V(t, x) \leq \frac{C}{(1+|x|)^{2+\delta}}, \quad x \in \mathbb{R}^{n}, \quad n \geq 3
$$

for some $C, \delta>0$. Then we can prove that $V(t, x)$ satisfies the assumptions of Theorem 1.2 and hence the local Strichartz estimates hold (and also the global ones, under the additional assumption of smallness at infinity).

Indeed, let $W(x)=V\left(t_{0}, x\right)$ for an arbitrary fixed $t_{0}$; we must show that $W(x)$ is of Strichartz type. The existence part of the definition is trivial; let us prove the estimates. Consider the operator $H=-\Delta+W(x) ; H$ has a unique selfadjoint extension by standard results, with spectrum contained in $[0,+\infty$ [; by Theorem XIII.58 in 14 $H$ has no strictly positive eigenvalues, since $W$ is bounded and decays as $|x|^{-2-\delta}$ at infinity; 0 is certainly not an eigenvalue since $H f=0$ implies $f=0$ easily. This implies that the operator $H$ has a purely continuous spectrum. Now Theorem 1.4 in [15] states that $P_{c} e^{i t H}$ satisfies the full set of Strichartz estimates when the potential is bounded and decays faster than $|x|^{-2}$ at infinity; here $P_{c}$ is the projection on the continuous subspace of $L^{2}$ for $H$, which coincides with all of $L^{2}$ as we have just proved. In conclusion, $W(x)=V\left(t_{0}, x\right)$ is of Strichartz type as claimed.

Remark 1.5. Condition (1.12) is quite natural, in view of the following argument: the standard rescaling $u_{\epsilon}(t, x)=u\left(\epsilon^{2} t, \epsilon x\right)$ takes equation (1.3) into the equation

$$
i \partial_{t} u_{\epsilon}-\Delta u_{\epsilon}+V_{\epsilon}(t, x) u_{\epsilon}=0, \quad V_{\epsilon}(t, x)=\epsilon^{2} V\left(\epsilon^{2} t, \epsilon x\right),
$$

and we have

$$
\left\|V_{\epsilon}\right\|_{L^{r} L^{s}}=\epsilon^{2\left(1-\frac{1}{r}-\frac{n}{2 s}\right)}\|V\|_{L^{r} L^{s}}
$$

so that the $L^{r} L^{s}$ norm of $V_{\epsilon}$ is independent of $\epsilon$ precisely when $r, s$ satisfy (1.12).

Indeed, by a suitable use of rescaling arguments, it is possible to show that the condition $1 / r+$ $n /(2 s)=1$ is necessary in order that the global Strichartz estimates be true for any potential belonging to the classes $L^{r} L^{s}$ (see Theorem 1.3 below).

Concerning the local Strichartz estimates, the situation is more interesting. When $1 / r+n /(2 s)<1$, as already observed in Remark 1.3 the local Strichartz estimates are an elementary consequence of Theorem 1.1 On the other hand, when $1 / r+n /(2 s)>1$, it is possible to show that the local Strichartz estimates fail. This case is more delicate; actually it is not even clear if equation (1.3) is well posed in $L^{2}$ under this assumption on $V$.

We collect our counterexamples in the following theorem, concerning the homogeneous equation

$$
i u_{t}-\Delta u+V(t, x) u=0 .
$$

Note that the case $(r, s)=(\infty, n / 2)$ is almost trivial since it is based on the construction of a standing wave for (1.25); we state it in some length both for completeness, and because the remaining counterexamples are based on it. Thus, in the proof of Theorem 1.3 it is essential to use potentials which change sign.

Theorem 1.3. Let $n \geq 2$. Then we have the following.

(i) (Case $r=\infty$ ) We can construct a potential $W(x) \in C_{0}^{\infty}\left(\mathbb{R}^{n}\right)$ and a function $u_{0} \in H^{s}$ for all $s>0$ such that

$$
-\Delta u_{0}+W(x) u_{0}+u_{0}=0 .
$$

Hence the function $u(t, x)=e^{-i t} u_{0}(x) \in C L^{2}$ solves (1.25) with

$$
V(t, x) \equiv W(x) \in L^{\infty}\left(\left[0,+\infty\left[; L^{n / 2}\left(\mathbb{R}^{n}\right)\right),\right.\right.
$$


and does not belong to the space $L^{p}\left(\left[0,+\infty\left[; L^{q}\right)\right.\right.$ for all admissible pairs $(p, q) \neq(\infty, 2)$. In other words, there exists a potential $V(t, x)$ belonging to $L^{\infty} L^{s}$ for all $s \in[1, \infty]$ such that the global Strichartz estimates (1.13) on $I=[0,+\infty[$ do not hold for equation (1.25).

(ii) (Counterexamples to global estimates) For every pair $(r, s)$ with $r \in[1, \infty[, s \in] n / 2, \infty]$ and

$$
\frac{1}{r}+\frac{n}{2 s} \neq 1
$$

we can construct a potential $V(t, x) \in L^{r}\left(\left[0,+\infty\left[; L^{s}\right)\right.\right.$ and a sequence of solutions $u_{k}(t, x) \in$ $C\left(\left[0,+\infty\left[; L^{2}\right)\right.\right.$ to equation (1.25) such that

$$
\lim _{k \rightarrow \infty} \frac{\left\|u_{k}\right\|_{L^{p} L^{q}}}{\left\|u_{k}(0)\right\|_{L^{2}}}=\infty \quad \text { for every admissible pair } \quad(p, q) \neq(\infty, 2) .
$$

(iii) (Counterexamples to local estimates) For every pair $(r, s)$ with $r \in[1, \infty[, s \in] n / 2, \infty]$ and

$$
\frac{1}{r}+\frac{n}{2 s}>1
$$

we can construct, on any given bounded time interval $I=[0, T]$, a potential $V(t, x) \in L^{r}\left([0, T] ; L^{s}\right)$ and a sequence of solutions $u_{k}(t, x) \in C\left([0, T] ; L^{2}\right)$ to equation (1.25) such that

$$
\lim _{k \rightarrow \infty} \frac{\left\|u_{k}\right\|_{L_{I}^{p} L^{q}}}{\left\|u_{k}(0)\right\|_{L^{2}}}=\infty \quad \text { for every admissible pair } \quad(p, q) \neq(\infty, 2) .
$$

We conclude the paper with a result showing that, at least for a restricted range of indices $r, s$, the conclusion of Theorem 1.3 part (iii), can be improved in an essential way. While the above theorem was based on suitable rescaling arguments, Proposition 1.4 exploits the pseudoconformal invariance of the Schrödinger equation.

Proposition 1.4. Let $n \geq 2$, and assume $r \in[1, \infty[$ and $s \in] n / 2, n[$ satisfy

$$
\frac{1}{2 r}+\frac{n}{2 s}>1
$$

Then we can construct a potential $V(t, x) \in L^{r}\left(0,1 ; L^{s}\left(\mathbb{R}^{n}\right)\right)$ and a solution $u(t, x) \in C\left([0,1] ; L^{2}\right)$ to equation (1.25) such that, for all admissible pairs $(p, q)$ with $p<\infty$, and for any $0<T<1$, we have

$$
u \in L^{p}\left(0, T ; L^{q}\left(\mathbb{R}^{n}\right)\right) \quad \text { but } \quad u \notin L^{p}\left(0,1 ; L^{q}\left(\mathbb{R}^{n}\right)\right) .
$$

\section{Proof of Theorem 1.1}

We shall consider in detail only the case $n \geq 3$; in the case $n=2$, when the endpoint fails, it is sufficient to replace in the following arguments the space $L_{J}^{2} L^{\frac{2 n}{n-2}}$ with any $L_{J}^{p} L^{q}$ with $q$ arbitrarily large.

We distinguish two cases, according to the value of $r \in[1, \infty[$.

2.1. Case A: $r \in[2, \infty[$. Consider a small interval $J=[0, \delta]$, and let $Z$ be the Banach space

$$
Z=C_{J} L^{2} \cap L_{J}^{2} L^{\frac{2 n}{n-2}}, \quad\|v\|_{Z}:=\max \left\{\|v\|_{L_{J}^{\infty} L^{2}},\|v\|_{L_{J}^{2} L^{\frac{2 n}{n-2}}}\right\} .
$$

Notice that, by interpolation, $Z$ is embedded in all admissible spaces $L_{J}^{p} L^{q}$.

For any $v(t, x) \in Z$ we define the mapping

$$
\Phi(v)=e^{i t \Delta} u_{0}+\int_{0}^{t} e^{i(t-s) \Delta}[F(s)-V(s) v(s)] d s .
$$

A direct application of (1.7), (1.8) gives

$$
\|\Phi(v)\|_{L_{J}^{p} L^{q}} \leq C_{0}\left\|u_{0}\right\|_{L^{2}}+C_{0}\|V v\|_{L_{J}^{p_{0}^{\prime}} L^{q_{0}^{\prime}}}+C_{0}\|F\|_{L_{J}^{\tilde{p}^{\prime}} L^{\tilde{q}^{\prime}}}
$$


for all admissible $(p, q),\left(p_{0}, q_{0}\right),(\tilde{p}, \tilde{q})$, and by Hölder's inequality we can write

$$
\|\Phi(v)\|_{L_{J}^{p} L^{q}} \leq C_{0}\left\|u_{0}\right\|_{L^{2}}+C_{0}\|V\|_{L_{J}^{r} L^{s}}\|v\|_{L_{J}^{2} L^{\frac{2 n}{n-2}}}+C_{0}\|F\|_{L_{J}^{\tilde{p}^{\prime}} L^{\tilde{q}^{\prime}}}
$$

provided we choose $p_{0}, q_{0}$ such that

Note that

$$
\frac{1}{p_{0}}=\frac{1}{2}-\frac{1}{r}, \quad \frac{1}{q_{0}}=\frac{n+2}{2 n}-\frac{1}{s} .
$$

$$
\frac{1}{p_{0}}+\frac{n}{2 q_{0}}=\frac{1}{2}+\frac{n+2}{4}-\left(\frac{1}{r}+\frac{n}{2 s}\right) \equiv \frac{1}{2}+\frac{n+2}{4}-1 \equiv \frac{n}{4}
$$

by our assumptions on $r, s$, and moreover

$$
r \in\left[2, \infty\left[\quad \Longrightarrow \quad p_{0} \in[2, \infty[\right.\right.
$$

so that our choice of $p_{0}, q_{0}$ always gives an admissible pair in the case under consideration.

In particular, choosing $(p, q)=(\infty, 2)$ or $(2,2 n /(n-2))$, we obtain

$$
\|\Phi(v)\|_{Z} \leq C_{0}\left\|u_{0}\right\|_{L^{2}}+C_{0}\|V\|_{L_{J}^{r} L^{s}}\|v\|_{Z}+C_{0}\|F\|_{L_{J}^{\tilde{p}^{\prime}} L^{\tilde{q}^{\prime}}}
$$

Thus $\Phi(v)$ belongs to all the admissible spaces $L_{J}^{p} L^{q}$, and to prove that $\Phi(v)$ belongs to $Z$ it remains only to show that $u$ is continuous with values in $L^{2}$. But this is an immediate consequence of the following simple remark:

Remark 2.1. Let $G(t, x) \in L_{J}^{a^{\prime}} L^{b^{\prime}}$ with $(a, b)$ admissible. Then the function

$$
w(t, x)=\int_{0}^{t} e^{i(t-s) \Delta} G(s) d s
$$

belongs to $C_{J} L^{2}$. Indeed, this is certainly true if we know in addition that $G$ is a smooth function, compactly supported in $x$ for each $t$. If we approximate $G$ by a sequence of such functions $G_{j}$ in the $L_{J}^{a^{\prime}} L^{b^{\prime}}$ norm, the Strichartz estimates imply that the corresponding functions $w_{j}$ converge in $L^{\infty} L^{2}$, whence the claim follows.

We have thus constructed a mapping $\Phi: Z \rightarrow Z$. Assume now the length $\delta$ of the interval $J$ is chosen so small that

$$
C_{0}\|V\|_{L_{J}^{r} L^{s}} \leq \frac{1}{2}
$$

this is certainly possible since $r<\infty$. With this choice we obtain immediately two consequences: first of all, the mapping $\Phi$ is a contraction on $Z$ and hence has a unique fixed point $v(t, x)$ which is the required solution; second, $v$ satisfies

$$
\|v\|_{L_{J}^{p} L^{q}} \leq C_{0}\left\|u_{0}\right\|_{L^{2}}+\frac{1}{2}\|v\|_{L_{J}^{p} L^{q}}+C_{0}\|F\|_{L_{J}^{\tilde{p}^{\prime}} L^{\tilde{q}^{\prime}}}
$$

whence we obtain

$$
\|v\|_{L_{J}^{p} L^{q}} \leq 2 C_{0}\left\|u_{0}\right\|_{L^{2}}+2 C_{0}\|F\|_{L_{J}^{\tilde{p}^{\prime}} L^{\tilde{q}^{\prime}}}
$$

It is clear that the above argument applies on any subinterval $J=\left[t_{0}, t_{1}\right] \subseteq I$ on which a condition like (2.5) holds; of course, we will obtain an estimate of the form

$$
\|v\|_{L_{J}^{p} L^{q}} \leq 2 C_{0}\left\|v\left(t_{0}\right)\right\|_{L^{2}}+2 C_{0}\|F\|_{L_{J}^{\tilde{p}^{\prime}} L^{\tilde{q}^{\prime}}}
$$

Notice also that 2.8) implies in particular

$$
\left\|v\left(t_{1}\right)\right\|_{L^{2}} \leq 2 C_{0}\left\|v\left(t_{0}\right)\right\|_{L^{2}}+2 C_{0}\|F\|_{L_{J}^{\tilde{p}^{\prime}} L^{\tilde{q}^{\prime}}}
$$

Now we can partition the interval $I$ (bounded or unbounded) in a finite number of subintervals on which condition (2.5) holds. Applying inductively the estimates (2.8) and (2.9) we easily obtain (1.13) and the claimed estimate for the Strichartz constant. 
The last remark (1.14) concerning the conservation of energy can be proved by approximation as follows: let $V_{j}(t, x)$ be a sequence of real valued smooth potentials, compactly supported in $x$, and let $v_{j}$ be the corresponding solutions; then the differences $w_{j}=v-v_{j}$ satisfy (in suitable integral sense)

$$
i \partial_{t} w_{j}-\Delta w_{j}+V w_{j}=\left(V-V_{j}\right) v_{j} \equiv F_{j} .
$$

Now we observe that the smooth solutions $v_{j}$ have a conserved energy; moreover, we can choose the approximating potentials $V_{j}$ in such a way that they converge to $V$ in $L_{I}^{r} L^{s}$ and their Strichartz constants do not exceed the above constructed constant for $V$. Indeed, if we can partition $I$ in a finite set of subintervals satisfying (2.5), we can choose exactly the same subintervals for each $V_{j}$ provided we construct $V_{j}$ by a convolution with standard mollifiers, so that their Lebesgue norm does not increase. In conclusion, the $v_{j}$ satisfy uniform Strichartz estimates, and this implies that the nonhomogeneous terms $F_{j}=\left(V-V_{j}\right) v_{j}$ tend to 0 in the (dual) admissible spaces, by estimates identical to the above ones. Thus in particular $w_{j} \rightarrow 0$ in $L^{\infty} L^{2}$ and this shows that also $v(t, x)$ satisfies the conservation of energy.

2.2. Case B: $r \in[1,2]$. The method in this case is quite similar to the above one, but instead of (2.2) we use the estimate

$$
\|\Phi(v)\|_{L_{J}^{p} L^{q}} \leq C_{0}\left\|u_{0}\right\|_{L^{2}}+C_{0}\|V v\|_{L_{J}^{r} L^{\frac{2 s}{s+2}}}+C_{0}\|F\|_{L_{J}^{\tilde{p}^{\prime}} L^{\tilde{q}^{\prime}}}
$$

where $(p, q)$ and $(\tilde{p}, \tilde{q})$ are arbitrary admissible pairs, while the pair $(r, 2 s /(s+2))$ is the dual of $\left(r^{\prime}, 2 s /(s-2)\right)$ and this last pair is admissible since

$$
\frac{1}{r^{\prime}}+\frac{n}{2} \cdot \frac{s-2}{2 s}=\frac{n}{2 s}+\frac{n}{2} \cdot \frac{s-2}{2 s}=\frac{n}{4}
$$

where we have used the assumption $1 / r+n /(2 s)=1$; notice also that $r \in[1,2]$ and hence $2 s /(s+2) \in[1,2]$ too.

Thus by Hölder's inequality we obtain

$$
\|\Phi(v)\|_{L_{J}^{p} L^{q}} \leq C_{0}\left\|u_{0}\right\|_{L^{2}}+C_{0}\|V\|_{L_{J}^{r} L^{s}}\|v\|_{L_{J}^{\infty} L^{2}}+C_{0}\|F\|_{L_{J}^{\tilde{p}^{\prime}} L^{\tilde{q}^{\prime}}}
$$

and choosing $(p, q)=(\infty, 2)$ or $(2,2 n /(n-2))$ and proceeding as above we arrive at

$$
\|\Phi(v)\|_{Z} \leq C_{0}\left\|u_{0}\right\|_{L^{2}}+\frac{1}{2}\|v\|_{Z}+C_{0}\|F\|_{L_{J}^{\tilde{p}^{\prime}} L^{\tilde{q}^{\prime}}} .
$$

From this point on, the proof is identical to the first case.

\section{Proof of Theorem 1.2}

The proof follows the same lines as the preceding one; indeed, the continuity in time of the potential allows to consider $V(t, x)$ as a small perturbation of $V\left(t_{0}, x\right)$ for $t$ near $t_{0}$.

Let $J=[0, \delta]$ be a small interval, and consider again the space

$$
Z=C_{J} L^{2} \cap L_{J}^{2} L^{\frac{2 n}{n-2}}, \quad\|v\|_{Z}:=\max \left\{\|v\|_{L_{J}^{\infty} L^{2}},\|v\|_{L_{J}^{2} L^{\frac{2 n}{n-2}}}\right\} .
$$

On $Z$ we construct a map $\Phi$ defined as follows:

$$
\Phi(v)=e^{i t H} u_{0}+\int_{0}^{t} e^{i(t-s) H}[F(s)-W(s) v(s)] d s,
$$

where

$$
H=\Delta-V(0, x), \quad W(t, x)=V(t, x)-V(0, x) .
$$


We have used the assumption that $V(0, x)$ is of Strichartz type (Definition 1.2) to make meaningfull the operators $e^{i t H}$; on the other hand this implies also that the full Strichartz estimates (1.7), (1.8) hold for the group $e^{i t H}$, hence we can write

$$
\|\Phi(v)\|_{L_{J}^{p} L^{q}} \leq C\left\|u_{0}\right\|_{L^{2}}+C\|W v\|_{L_{J}^{2} L^{\frac{2 n}{n+2}}}+C\|F\|_{L_{J}^{\tilde{p}^{\prime}} L^{\tilde{q}^{\prime}}}
$$

for all admissible pairs $(p, q)$ and $(\tilde{p}, \tilde{q})$. Notice that here $C$ is a constant depending on $V$ and the interval $J$ only, and can be assumed to be non increasing when $\delta \downarrow 0$. This implies

$$
\|\Phi(v)\|_{L_{J}^{p} L^{q}} \leq C\left\|u_{0}\right\|_{L^{2}}+C\|W\|_{L_{J}^{\infty} L^{n / 2}}\|v\|_{L_{J}^{2} L^{\frac{2 n}{n-2}}}+C\|F\|_{L_{J}^{\tilde{p}^{\prime}} L^{\tilde{q}^{\prime}}}
$$

and if $\delta$ is so small that

$$
C\|W\|_{L_{J}^{\infty} L^{n / 2}} \leq \frac{1}{2}
$$

which is possible by the continuity of $V(t, x)$ as an $L^{n / 2}$-valued function, we arrive at

$$
\|\Phi(v)\|_{Z} \leq C\left\|u_{0}\right\|_{L^{2}}+\frac{1}{2}\|v\|_{Z}+C\|F\|_{L_{J}^{\tilde{p}^{\prime}} L^{\tilde{q}^{\prime}}}
$$

This guarantees, as above, the existence of a unique local solution belonging to the space $Z$ and satisfying the Strichartz estimates with some constant $C(0)$ for some time interval $[0, \delta)$.

The same argument can be applied near each point $t_{0} \in I$. More precisely, let $J=\left[t_{0}-\delta, t_{0}+\delta\right] \cap I$ and assume $\delta>0$ is so small that the potential

$$
W(t, x)=V(t, x)-V\left(t_{0}, x\right)
$$

satisfies

$$
\|W(t, \cdot)\|_{L^{n / 2}} \leq\left(2 C\left(V\left(t_{0}, x\right)\right)\right)^{-1} \quad \text { for } t \in J,
$$

where $C\left(V\left(t_{0}, x\right)\right)$ is the Strichartz constant corresponding to the potential $V\left(t_{0}, x\right)$ and relative to the interval $\left[0, t_{0}+1\right]$. Then we may argue as above, and we obtain that for any given initial time $t_{1} \in J$ and for any $f \in L^{2}$, the Cauchy problem

$$
i \partial_{t} u-H u=F(t, x)-W(t, x) u, \quad u\left(t_{1}\right)=f, \quad H=\Delta-V\left(t_{0}, x\right)
$$

(interpreted as usual in integral form via the group $e^{i t H}$ ) has a unique solution in $Z=C_{J} L^{2} \cap$ $L_{J}^{2} L^{\frac{2 n}{n-2}}$, which satisfies the Strichartz estimates

$$
\|\Phi(v)\|_{Z} \leq 2 C\left(t_{0}\right)\left\|u_{0}\right\|_{L^{2}}+2 C\left(t_{0}\right)\|F\|_{L_{J}^{\tilde{p}^{\prime}} L^{\tilde{q}^{\prime}}}
$$

for some constant $C\left(t_{0}\right)$ depending on the point $t_{0}$ but not on the initial time $t_{1} \in J$.

Now we may proceed by a continuation argument as follows. Extend the local solution constructed on $[0, \delta]$ to a maximal interval $\left[0, T^{*}\right.$; i.e., consider the union of all intervals $[0, \delta]$ on which a solution $u \in C\left([0, \delta] ; L^{2}\right) \cap L^{2}\left(0, \delta ; L^{\frac{2 n}{n-2}}\right)$ exists and satisfies (for all admissible pairs) the Strichartz estimates with some constant $C_{\delta}$. Assume by contradiction that $T^{*}<T$. Then the above local argument applied at $t_{0}=T^{*}$ on a suitable interval of the form $J=\left[T^{*}-\varepsilon, T^{*}+\varepsilon\right]$ shows that we can patch the maximal solution and extend it to $\left[0, T^{*}+\varepsilon\right]$. Moreover, we claim that the extended solution satisfies the Strichartz estimates on $\left[0, T^{*}+\varepsilon\right]$ : indeed, chosen any $t_{1}$ such that $T^{*}-\varepsilon<t_{1}<T^{*}$, by construction we see that the estimates hold both on $I_{1}=\left[0, t_{1}\right]$, with initial data at $t=0$ :

$$
\|u\|_{L_{I_{1}}^{p} L^{q}} \leq C^{\prime}\left\|u\left(t_{0}\right)\right\|_{L^{2}}+C^{\prime}\|F\|_{L_{I_{1}}^{\tilde{p}^{\prime}} L^{\tilde{q}^{\prime}}}
$$

and on $J=\left[T^{*}-\varepsilon, T^{*}+\varepsilon\right]$, with initial data at $t=t_{1}$ :

$$
\|u\|_{L_{J}^{p} L^{q}} \leq C^{\prime}\left\|u\left(t_{1}\right)\right\|_{L^{2}}+C^{\prime}\|F\|_{L_{J}^{\tilde{p}^{\prime}} L^{\tilde{q}^{\prime}}}
$$

for a suitable constant $C^{\prime}$. Since $\left\|u\left(t_{1}\right)\right\|_{L^{2}}$ can be estimated exactly by (3.9) $(p=2, q=\infty)$, we easily conclude the proof of our claim. This contradicts the assumption $T^{*}<T$ and we obtain that $T^{*}=T$. 
The modifications required to prove the final remark concerning the case $I=[0, \infty[$, and also Remark 1.4 are obvious.

\section{Proof of the COUNTEREXAMPLES}

4.1. An eigenvalue problem. The first step in our construction requires to find a potential $V(x)$ such that the operator $-\Delta+V(x)$ has a negative eigenvalue, i.e., such that the equation

$$
-\Delta u_{0}+V(x) u_{0}+\gamma^{2} u_{0}=0
$$

admits a solution $u_{0} \in H^{1}$ for some $\gamma>0$. There are many results on this problem, and in general there is a clear connection between the number of such eigenvalues and the size of the negative part of $V$, in a suitable norm. This is true both in the negative sense (explicit bounds on the number of the eigenvalues) and in the positive sense, which is our main focus here. For instance, it is known that (see 14]) if $V(x) \in L^{n / 2}\left(\mathbb{R}^{n}\right)$ satisfies the assumption

$$
\text { the set }\left\{x \in \mathbb{R}^{n}: V(x)<0\right\} \text { has a positive measure, }
$$

then there exists $\lambda_{0}>0$ such that, for all $\lambda>\lambda_{0}$, the equation

$$
-\Delta u_{0}+\lambda V(x) u_{0}+\gamma^{2} u_{0}=0
$$

admits at least a solution $f \in H^{1}$ for some $\gamma>0$. It can also be proved that the dimension of the eigenspace grows to infinity as $\lambda$ tends to infinity.

However, for our purposes here we need only a much less precise result, which can be proved directly by an elementary variational argument. Indeed, take any smooth compactly supported function $w(x)$ such that $w\left(x_{0}\right)>0$ at least in one point $x_{0}$. Then consider the minimization problem with a constraint

$$
\min _{f \in M} \int_{\mathbb{R}^{n}}\left(|\nabla f|^{2}+|f|^{2}\right) d x \quad \text { on } \quad M=\left\{f \in H^{1}: \int_{\mathbb{R}^{n}} w(x)|f|^{2} d x=1\right\} .
$$

Note that $M$ is not empty, thanks to the assumption $w\left(x_{0}\right)>0$. The existence of a solution to problem (4.4) can be proved easily by a standard compactness argument, since we can work on the (bounded) support of $w(x)$. On the other hand, the Euler-Lagrange equation of the problem is

$$
-\Delta f+f=\mu w(x) f
$$

(where $\mu$ is a Lagrange multiplier); hence, choosing $W(x)=-\mu w(x)$ and $u_{0}=f$, we see that $u_{0}$ solves the equation

$$
-\Delta u_{0}+W(x) u_{0}+u_{0}=0
$$

and hence

$$
u(t, x)=e^{-i t} u_{0}(x) \text { solves } i u_{t}-\Delta u+W(x) u=0 .
$$

Note also that a trivial bootstrapping argument gives $u_{0} \in H^{s}$ for all $s>0$.

This concludes the proof of Theorem 1.3 part (i).

4.2. Proof of Theorem 1.3, case $1 / r+n /(2 s)<1, r \neq \infty$. We start from the function (4.7) and we apply the standard rescaling

$$
u(t, x) \mapsto u_{\epsilon}(t, x)=u\left(\epsilon^{2} t, \epsilon x\right) \equiv e^{-i \epsilon^{2} t} u_{0}(\epsilon x) .
$$

Then the function $u_{\epsilon}$ solves globally

$$
i \partial_{t} u_{\epsilon}-\Delta u_{\epsilon}+W_{\epsilon}(x) u_{\epsilon}=0, \quad W_{\epsilon}(x)=\epsilon^{2} W(\epsilon x) .
$$

Consider now two monotone sequences of positive real numbers

$$
0=T_{0}<T_{1}<\cdots<T_{k} \uparrow+\infty, \quad 0<\epsilon_{k} \downarrow 0, \quad k=0,1,2,3, \ldots
$$


and define a potential $V(t, x)$ on $\left[0,+\infty\left[\times \mathbb{R}^{n}\right.\right.$ by patching the potentials $V_{\epsilon}$ as follows:

$$
V(t, x)=W_{\epsilon_{k}}(x) \quad \text { for } \quad t \in\left[T_{k}, T_{k+1}[, \quad k=0,1,2, \ldots\right.
$$

Thus $u_{\epsilon_{k}}$ solves the equation

$$
i \partial_{t} u-\Delta u+V(t, x) u=0
$$

on the interval $\left[T_{k}, T_{k+1}[\right.$.

Choose now $r$ and $s$ satisfying

$$
\frac{1}{r}+\frac{n}{2 s}<1, \quad r \neq \infty,
$$

and assume we can choose the parameters $T_{k}, \epsilon_{k}$ in such a way that

$$
\|V\|_{L^{r} L^{s}} \leq\|W\|_{L^{s}} \sum_{k \geq 0}\left(T_{k+1}-T_{k}\right)^{1 / r} \epsilon^{2-n / s}<\infty,
$$

then $V(t, x) \in L^{r}\left(\left[0,+\infty\left[; L^{s}\right)\right.\right.$. On the other hand by Theorem 1.1 we can extend (uniquely) $u_{\epsilon_{k}}$ to a global solution of (4.12) in $C\left(\left[0,+\infty\left[; L^{2}\left(\mathbb{R}^{n}\right)\right)\right.\right.$ which we shall denote by $u_{k}(t, x)$. Notice that, by the same theorem, we have

$$
\left\|u_{k}(t, \cdot)\right\|_{L^{2}} \equiv \text { const. } \equiv\left\|u_{\epsilon_{k}}\left(T_{k}\right)\right\|_{L^{2}} \equiv \epsilon^{-n / 2}\left\|u_{0}\right\|_{L^{2}}
$$

recalling the explicit expression (4.8) of $u_{\epsilon}$. On the other hand, we can write

$$
\left\|u_{k}\right\|_{L^{p}\left(\mathbb{R} ; L^{q}\right)} \geq\left\|u_{k}\right\|_{L^{p}\left(T_{k}, T_{k+1} ; L^{q}\right)} \equiv\left\|u_{\epsilon_{k}}\right\|_{L^{p}\left(T_{k}, T_{k+1} ; L^{q}\right)} \equiv\left(T_{k}-T_{k+1}\right)^{1 / p} \epsilon_{k}^{-n / q}\left\|u_{0}\right\|_{L^{q}}
$$

by an elementary calculation. The Strichartz esimates are violated when

$$
\frac{\left\|u_{k}\right\|_{L^{p}\left(\mathbb{R} ; L^{q}\right)}}{\left\|u_{k}(0)\right\|_{L^{2}}} \text { is unbounded, }
$$

and this holds provided the parameters $T_{k}, \epsilon_{k}$ satisfy the condition

$$
\frac{\left\|u_{k}\right\|_{L^{p}\left(\mathbb{R} ; L^{q}\right)}}{\left\|u_{k}(0)\right\|_{L^{2}}} \geq \frac{\left\|u_{\epsilon_{k}}\right\|_{L^{p}\left(T_{k}, T_{k+1} ; L^{q}\right)}}{\left\|u_{\epsilon_{k}}(0)\right\|_{L^{2}}} \equiv \frac{\left\|u_{0}\right\|_{L^{q}}}{\left\|u_{0}\right\|_{L^{2}}}\left(T_{k}-T_{k+1}\right)^{1 / p} \epsilon_{k}^{\frac{n}{2}-\frac{n}{q}} \rightarrow \infty .
$$

In conclusion, we only need to adjust the parameters (4.10) so to satisfy the two conditions (4.14), 4.18):

$$
\sum_{k \geq 0}\left(T_{k+1}-T_{k}\right)^{1 / r} \epsilon_{k}^{2-n / s}<\infty, \quad\left(T_{k}-T_{k+1}\right)^{1 / p} \epsilon_{k}^{\frac{n}{2}-\frac{n}{q}} \rightarrow \infty,
$$

given an admissible pair $(p, q)$ with $p \neq \infty$ and $(r, s)$ as in (4.13). With the special choices

$$
T_{0}=0, \quad T_{k+1}=T_{k}+k^{\alpha}, \quad \epsilon_{0}=1, \quad \epsilon_{k}=k^{-\beta / 2}, \quad k=1,2,3, \ldots
$$

for some $\alpha, \beta>0$, the conditions reduce to

$$
\frac{\alpha}{r}+\beta \frac{n}{2 s}<\beta-1, \quad \frac{\alpha}{p}+\beta \frac{n}{2 q}>\beta \frac{n}{4} .
$$

Since $(p, q)$ is admissible, the second condition simplifies to $\alpha>\beta$, and rearranging the first one we are reduced to

$$
\frac{\alpha-\beta}{r}+\beta\left(\frac{1}{r}+\frac{n}{2 s}\right)<\beta-1, \quad \alpha>\beta .
$$

The term in brackets is smaller then 1 by assumption, hence if we choose any

$$
\alpha>\beta>\left[1-\left(\frac{1}{r}+\frac{n}{2 s}\right)\right]^{-1}
$$

with $\alpha$ close enough to $\beta$, we conclude the proof of the first part of Theorem [1.2 (ii). 
4.3. Proof of Theorem 1.3, case $1 / r+n /(2 s)>1, r \neq \infty$. As in case 4.2 the proof is based on a rescaling argument. First of all we prove part (ii). Consider again the rescaled solution (4.8) which solves equation (4.9) globally with a smooth compactly supported potential $W_{\epsilon}(x)=\epsilon^{2} W(\epsilon x)$. Now, take two monotone sequences of positive real numbers

$$
1=\epsilon_{0}<\epsilon_{1}<\cdots<\epsilon_{k} \uparrow+\infty, \quad 0<\delta_{k} \downarrow 0, \quad k=0,1,2,3, \ldots
$$

and define a potential $V(t, x)$ on $\left[0,+\infty\left[\times \mathbb{R}^{n}\right.\right.$ as follows:

$$
V(t, x)= \begin{cases}W_{\epsilon_{k}}(x) & \text { if } t \in\left[k, k+\delta_{k}\right], x \in \mathbb{R}^{n}, \\ 0 & \text { elsewhere. }\end{cases}
$$

Note that $V(t, x) \in L_{I}^{\infty} L^{\infty}$ for any bounded time interval $I$, while globally

$$
\|V\|_{L^{r} L^{s}} \leq\|W\|_{L^{s}} \sum_{k \geq 0} \delta_{k}^{1 / r} \epsilon_{k}^{2-n / s} .
$$

As above, the function $u_{\epsilon_{k}}$ solves the equation

$$
i \partial_{t} u-\Delta u+V(t, x) u=0
$$

on the interval $t \in\left[k, k+\delta_{k}\right]$, and can be extended to a global solution $u_{k}(t, x)$ of the same equation thanks to the existence part of Theorem 1.1] (recall that $V \in L_{I}^{\infty} L^{\infty}$ ). Moreover, $u_{k}$ has a conserved energy

$$
\left\|u_{k}(t, \cdot)\right\|_{L^{2}} \equiv\left\|u_{k}(k, \cdot)\right\|_{L^{2}} \equiv \epsilon^{-n / 2}\left\|u_{0}\right\|_{L^{2}} .
$$

Then, as before, we can estimate

$$
\frac{\left\|u_{k}\right\|_{L^{p}\left(\mathbb{R} ; L^{q}\right)}}{\left\|u_{k}(0)\right\|_{L^{2}}} \geq \frac{\left\|u_{\epsilon_{k}}\right\|_{L^{p}\left(k, k+\delta_{k} ; L^{q}\right)}}{\left\|u_{\epsilon_{k}}(0)\right\|_{L^{2}}} \equiv \frac{\left\|u_{0}\right\|_{L^{q}}}{\left\|u_{0}\right\|_{L^{2}}} \delta_{k}^{1 / p} \epsilon_{k}^{\frac{n}{2}-\frac{n}{q}} .
$$

Again, in order to violate the Stichartz estimates for an admissible couple $(p, q)$ and the potential $V \in L^{r} L^{s}$, it is sufficient to satisfy the two conditions

$$
\sum_{k \geq 0} \delta_{k}^{1 / r} \epsilon_{k}^{2-n / s}<\infty, \quad \delta_{k}^{1 / p} \epsilon_{k}^{\frac{n}{2}-\frac{n}{q}} \rightarrow \infty .
$$

With the special choices

$$
\delta_{k}=k^{-\alpha}, \quad \epsilon_{k}=k^{\beta / 2},
$$

the parameters $\alpha, \beta>0$ to be precised, we are reduced to

$$
-\frac{\alpha}{r}+\left(1-\frac{n}{2 s}\right) \beta<-1, \quad-\frac{\alpha}{p}+\left(\frac{n}{4}-\frac{n}{2 q}\right) \beta>0 .
$$

Since $(p, q)$ is an admissible pair, the second condition is equivalent to $\alpha<\beta$ and we can rewrite the conditions as

$$
\frac{\alpha-\beta}{r}+\left(\frac{1}{r}+\frac{n}{2 s}\right) \beta>\beta+1, \quad \alpha<\beta .
$$

Recall now that we are considering the case

$$
\frac{1}{r}+\frac{n}{2 s}>1,
$$

hence we may choose any $\beta$ such that

$$
\beta>\left[\left(\frac{1}{r}+\frac{n}{2 s}\right)-1\right]^{-1}
$$

and choosing then any $\alpha<\beta$ close enough to $\beta$, we easily satisfy the conditions (4.33).

This concludes the proof of part (ii) of Theorem 1.3 
Part (iii) can be proved by a simple modification of the preceding proof. Indeed, consider again the sequence $\delta_{k}=k^{-\alpha}$ constructed above, and notice that it is not restrictive to assume that $\beta>\alpha>1$. Thus the series $\sum \delta_{k}$ converges, and the sequence of partial sums

$$
T_{k}=\sum_{j=0}^{k} \delta_{k}
$$

is positive, strictly increasing, and converges to

$$
\lim _{k \rightarrow \infty} T_{k}=T \equiv \sum_{k \geq 0} \delta_{k}
$$

We can now modify the definition (4.25) of the potential $V(t, x)$ as follows:

$$
V(t, x)= \begin{cases}W_{\epsilon_{k}}(x) & \text { if } t \in\left[T_{k}, T_{k}+\delta_{k}\right], x \in \mathbb{R}^{n} \\ 0 & \text { if } t \in\left[0, \delta_{0}[.\right.\end{cases}
$$

This defines a potential on $I \times \mathbb{R}^{n}=[0, T] \times \mathbb{R}^{n}$, whose $L_{I}^{r} L^{s}$ is given again by 4.26). The remaining arguments of the preceding proof apply without modification.

The proof of Theorem 1.3 is concluded.

4.4. Proof of Proposition 1.4. The main tool of the proof is the pseudoconformal transform

$$
u(t, x) \quad \mapsto \quad U(T, X)=e^{-i \frac{|X|^{2}}{4 T}} T^{-\frac{n}{2}} u\left(-\frac{1}{T}, \frac{X}{T}\right)
$$

which takes a solution $u(t, x)$ of the Schrödinger equation in the variables $t, x$ into another solution of the same equation, in the variables $T, X$. If we apply the transform to the solution (4.7), we obtain a function $U(T, X)$ which solves

$$
i \partial_{T} U-\Delta_{X} U+V(T, X) U=0, \quad U(1, X)=e^{i-i|X|^{2}} u_{0}(X),
$$

where the potential $V(T, X)$ is given by

$$
V(T, X)=\frac{1}{T^{2}} W\left(\frac{X}{T}\right) .
$$

It is easy to compute explicitly the norm of $V$ on the interval $[0,1]$ :

$$
\|V\|_{L^{r}\left(\delta, 1 ; L^{s}\right)}=\left(\int_{\delta}^{1} T^{r(n / s-2)} d T\right)^{1 / r}\|W\|_{L^{s}}<\infty
$$

and this integral converges since our assumption (1.31) on the pair $(r, s)$ is equivalent to

$$
r\left(\frac{n}{s}-2\right)>-1
$$

On the other hand, the $L_{I}^{p} L^{q}$ norm of $U(T, X)$ on an interval of the form $[\delta, 1]$ with $0<\delta<1$ is given by

$$
\|U\|_{L_{I}^{p} L^{q}}=\left(\int_{\delta}^{1} T^{p\left(\frac{n}{q}-\frac{n}{2}\right)}\right)^{1 / p}\|W\|_{L^{q}} \equiv\left(\int_{\delta}^{1} T^{-2}\right)^{1 / p}\|W\|_{L^{q}}
$$

since admissible pairs $(p, q)$ satisfy $p(n / q-n / 2) \equiv-2$. This implies that $U(T, X)$ belongs ot all $L_{I}^{p} L^{q}$ spaces for $I=[\delta, 1]$ for all $0<\delta<1$, but not for $I=[0,1]$ where the integral diverges. Note also that

$$
\|U(1, \cdot)\|_{L^{2}} \equiv\left\|u_{0}\right\|_{L^{2}} .
$$

It is sufficient now to apply to $U(T, X)$ a reflection and a translation in time $T$ to obtain exactly the counterexample required in Theorem 1.4 The proof is concluded. 


\section{REFERENCES}

[1] J. Bourgain. Exponential sums and nonlinear Schrödinger equations. Geom. Funct. Anal., 3(2):157-178, 1993.

[2] J. Bourgain. Fourier transform restriction phenomena for certain lattice subsets and applications to nonlinear evolution equations. I. Schrödinger equations. Geom. Funct. Anal., 3(2):107-156, 1993.

[3] J. Bourgain. Global wellposedness of defocusing critical nonlinear Schrödinger equation in the radial case. $J$. Amer. Math. Soc., 12(1):145-171, 1999.

[4] N. Burq, P. Gérard, and N. Tzvetkov. Strichartz inequalities and the nonlinear Schrödinger equation on compact manifolds. Amer. J. Math., 126(3):569-605, 2004.

[5] T. Cazenave. Semilinear Schrödinger equations, volume 10 of Courant Lecture Notes in Mathematics. New York University Courant Institute of Mathematical Sciences, New York, 2003.

[6] O. Costin, R.D. Costin, A. Rokhlenko and J. Lebowitz. Evolution of a model quantum system under time periodic forcing: conditions for complete ionization. Comm. Math. Phys., 221(1):1-26, 2001.

[7] J. Ginibre and G. Velo. Generalized Strichartz inequalities for the wave equation. J. Funct. Anal., 133(1):50-68, 1995.

[8] V. Georgiev, A. Ivanov. Existence and mapping properties of the wave operator for the Schrödinger equation with singular potential. Preprint 2004.

[9] M. Goldberg and W. Schlag. Dispersive estimates for Schrödinger operators in dimensions one and three. Preprint 2003.

[10] J. Howland. Stationary scattering theory for time-dependent Hamiltonians. Math. Ann., 207: 315-335, 1974.

[11] J.-L. Journé, A. Soffer, and C. D. Sogge. Decay estimates for Schrödinger operators. Comm. Pure Appl. Math., 44(5):573-604, 1991.

[12] M. Keel and T. Tao. Endpoint Strichartz estimates. Amer. J. Math., 120(5):955-980, 1998.

[13] V. Pierfelice. Strichartz estimates for the Schrödinger and heat equations perturbed with singular and time dependent potentials. Preprint 2003.

[14] M. Reed and B. Simon. Methods of modern mathematical physics. IV. Analysis of operators. Academic Press [Harcourt Brace Jovanovich Publishers], New York, 1978.

[15] I. Rodnianski and W. Schlag. Time decay for solutions of Schrödinger equations with rough and time-dependent potentials. Invent. Math., 155(3):451-513, 2004.

[16] K. Yajima. Large time behaviors of time-periodic quantum systems. In Differential equations (Birmingham, Ala., 1983), volume 92 of North-Holland Math. Stud., pages 589-597. North-Holland, Amsterdam, 1984.

[17] K. Yajima. Existence of solutions for Schrödinger evolution equations. Communications in Mathematical Physics, 110(3):415-426, 1987. 